\title{
Corneal Cross-Linking and Safety Issues
}

\author{
Eberhard Spoerl ${ }^{*}$, Anne Hoyer, Lutz E. Pillunat and Frederik Raiskup
}

Department of Ophthalmology, Carl Gustav Carus University Hospital, Dresden, Germany

\begin{abstract}
Purpose: To compile the safety aspects of the corneal collagen cross-linking (CXL) by means of the riboflavin/UVA (370 $\mathrm{nm})$ approach.

Materials and Methodology: Analysis of the current treatment protocol with respect to safety during CXL.

Results: The currently used UVA dose density of $5.4 \mathrm{~J} / \mathrm{cm}^{2}$ and the corresponding irradiance of $3 \mathrm{~mW} / \mathrm{cm}^{2}$ are below the known damage thresholds of UVA for the corneal endothelium, lens, and retina. Regarding the photochemical damages due to the free radicals the damage threshold for endothelial cells is $0.35 \mathrm{~mW} / \mathrm{cm}^{2}$. In a $400 \mu \mathrm{m}$ thick corneal stroma saturated with riboflavin, the irradiance at the endothelial level is about $0.18 \mathrm{~mW} / \mathrm{cm}^{2}$, which is a factor of 2 smaller than the damage threshold.

Conclusion: As long as the corneal stroma treated has a minimal thickness of 400 microns (as recommended), neither corneal endothelium nor deeper structures such as lens and retina will suffer any damages. The light source should provide a homogenous irradiance avoiding hot spots.
\end{abstract}

Keywords: Keratoconus, biomechanics, cornea, ultraviolet light, cross-linking.

\section{INTRODUCTION}

From the beginning of the CXL research the safety of the treatment stood in the center of attention because this procedure was not applied in tissue engineering of isolated collagen structures. The aim was to develop a clinically applicable method for eyes in vivo to create additional chemical bonds inside the corneal stroma by means of a highly localized photopolymerization while minimizing exposure to the surrounding structures of the eye $[1,2]$. There were some points to taken under consideration:

- $\quad$ The duration of the treatment should not be too long.

- The transparency of the cornea should not be changed.

- The cross-linking effect should only include the cornea.

(Cross linking and damaging of adjacent tissue should be avoided).

It was a challenge in the optimizing of the treatment parameters to reach a sufficient biomechanical effect in the cornea and avoid damage of the endothelium, lens or retina. The treatment parameters should fullfil two requirements: the biomechanical effect and the safety. Many investigations were necessary for the fine-tuning of these treatment parameters [3-8].

\section{The Choosing of the Photochemical Cross-Linking}

There are two different collagen cross-linking methods: the chemical cross-linking uses solutions like glutaraldehyde,

*Address correspondence to this author at the Department of Ophthalmology, Carl Gustav Carus University Hospital, Fetscherstr. 74, D-01307 Dresden, Germany; Tel: +49 351458 3763; Fax: +49 351458 4335;

E-mail: eberhard.spoerl@uniklinikum-dresden.de transglutaminase, genepin and nitroalcohol, and the photooxidative (also called physical) cross-linking uses light especially UV-light [2]. The application of liquid crosslinker to the curved cornea is not easy and the diffusion of the liquid cannot be controlled during application. Thus, we decided to use the photooxitative cross-linking for a safer application $[3,9]$.

\section{The Choosing of the Treatment Parameters with Respect to Safety}

Singlet oxygen is necessary for the photooxidative crosslinking. Riboflavin is one of the most potent producers of these oxygen radicals. Riboflavin (vitamin B2) is not toxic and it is also used as a food dye. However, riboflavin is not only a photosensitizer - it acts also as a UV absorber. Since the UV light is effective only in the absorbed areas, it is desirable that the irradiation is absorbed in about $400 \mu \mathrm{m}$ thick corneal stroma tissue $[10,11]$.

This high absorption is achieved by the selection of the wavelength of the used UV light at $370 \mathrm{~nm}$, a wavelength that corresponds to one of the riboflavin's absorption maxima. For the absorption of ultraviolet light in the cornea the concentration of the superficially applied riboflavin solution of $0.1 \%$ and the time course of the diffusion process is relevant. Applied riboflavin must diffuse into the corneal stroma and this process requires certain time [11]. The intact epithelium acts as a barrier that inhibits the diffusion of riboflavin (molecular weight $376.37 \mathrm{~g} / \mathrm{mol}$ ) into the cornea $[3,12,13]$. For that reason the epithelium must be debrided from the intended treatment area because this simple procedure removes a diffusion barrier for the riboflavin molecule and speeds saturation of the corneal stromal tissue [3]. Then riboflavin diffuses through the cornea and a concentration gradient is formed. Though the highest concentration of riboflavin is reached in the anterior stroma 
[14], however after 20-30 min a sufficient concentration is reached also in the posterior stroma [11]. As a result only a small part of the UV penetrates through the cornea and thus deeper ocular structures like lens or retina are protected from the potential hazards of free radical formation. After the riboflavin has traversed the cornea, it enters into the anterior chamber. The aqueous humour without riboflavin does not have any relevant absorption at $370 \mathrm{~nm}$ but clinically it starts to stain after about $5 \mathrm{~min}$ of surface exposure to riboflavin. By means of slit lamp inspection using blue light the surgeon has to assure that riboflavin has appeared in the anterior chamber before the UV-irradiation has started [15]. The yellow staining of the anterior chamber serves as a safety feature, indicating that the riboflavin has penetrated the cornea and the cornea is thoroughly saturated. Only a circular area of $8 \mathrm{~mm}$ in diameter is then exposed to UVlight with a wavelength of $370 \pm 5 \mathrm{~nm}$ and an irradiance of 3 $\mathrm{mW} / \mathrm{cm}^{2}$ for a total time of $30 \mathrm{~min}$. This corresponds to a total dose of $3.4 \mathrm{~J}(\mathrm{~J}=\mathrm{Joule})$ or a total dose density of 5.4 $\mathrm{J} / \mathrm{cm}^{2}$ to the cornea. Lambert-Beer's law yields a reduction of the irradiance due to absorption in a $400 \mu \mathrm{m}$-thick stroma satured with riboflavin (Fig. 1). Therefore, due to the additional riboflavin shielding all structures behind the corneal stroma including the cornea endothelium, anterior chamber, iris, lens, and retina are theoretically exposed to a residual UV-dose density that is less than $1 \mathrm{~J} / \mathrm{cm}^{2}$ as recommended by the UV-guidelines $[11,16]$.

The aim in the biomechnical effect is to reach a stiffness of the keratoconic cornea similar to the normal one. The stiffness of the keratoconic cornea is about $70 \%$ of a healthy cornea [17]. It was not our aim to harden the cornea extremly and to convert it in a non-physiological range. For that reason we choose a low irradiance of $3 \mathrm{~mW} / \mathrm{cm}^{2}$ for 30 minutes. Thus, we increase the stability of the cornea and the cross-linked collagen network allowing keratocyte repopulation [18, 19]. The strong cross-linked collagen inhibits the repopulation of cells [20].

\section{Design of a Light Source with Respect to Safety}

All the above safety considerations are based on an irradiance that is homogenous within the field of UVAapplication. However, if optical inhomogenities such as hot spots are present the damage thresholds may be exceeded locally leading to localized endothelial damage although the average irradiance may be less than $3 \mathrm{~mW} / \mathrm{cm}^{2}$. Therefore, clinically used light sources must guarantee a perfect homogenity of the irradiance across the beamed area.

The UV illumination system was designed based on ultraviolet LEDs with a peak emmitance at $365 \mathrm{~nm}$. A beam path according to Koehler [21] is focused through a variable diaphragm onto the corneal surface with a special beam homogenizing micro structure [22] in order to ensure homogenity of illumination on the cornea. In the optical design according to Koehler the UV light diaphragm is imaged onto the corneal front surface and as a consequence is the UV light that is focused on the front surface of the cornea strongly scattered in the ocular media behind the cornea. Thus the safety considerations are fully applied and the real radiant exposures in the eye are probably even lower. In order to obtain an estimate of the real intraocular illumination intensities during treatments using the presented homogenizing UV light source, the radiant exposure was experimentally measured in the retinal plane of porcines eyes [23].

\section{Prevention of the Corneal Endothelium Damage}

The photopolymerization process inducing additional cross-links in the corneal stroma is carried out by free radicals mediated by the riboflavin irradiated with UV-light. Such radicals can create cell damages that may be tolerable in keratocytes population but not in the corneal endothelium. The cytotoxicity of the riboflavin/UVA treatment on keratocytes and endothelium cells was studied by Wollensak and coworkers and the cytotoxic threshold of the UVA/Riboflavin for endothelial cells and keratocytes was

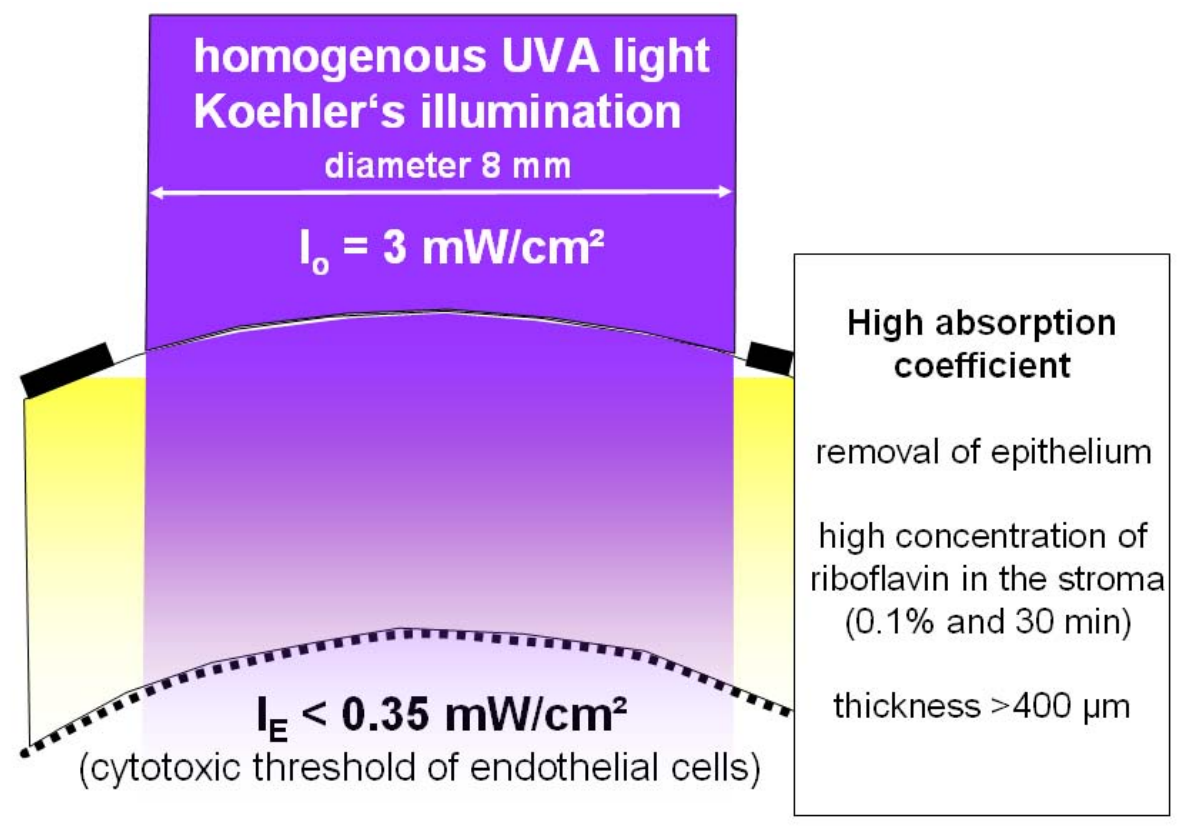

Fig. (1). Safety aspects of corneal cross-linking with riboflavin and UVA. 
determined [4-7]. The cell population that is the most critical to suffer from damages either from UV-light directly or from the free radicals is the corneal endothelium because it is immediately adjacent to the corneal stroma and these cells in the normal human cornea have low regenerative capacity. With the above mentioned treatment parameters the cytotoxic threshold of the endothelial cells is not reached. Clinical studies provide the best evidence. By means of confocal microscopy in keratoconus eyes treated with CXL no pathologic signs of the endothelial damage were detected $[24,25]$. In other clinical studies using the recommended treatment parameters there was no change in the endothelial cell count observed after CXL [9, 26, 27].

\section{CONCLUSION}

In summary, regarding the safety issues, procedure should respect the following criteria: (1) to facilitate diffusion of riboflavin the epithelium should be removed; (2) a $0.1 \%$ riboflavin solution must be applied for 30 minutes before UV-exposure, during this treatment riboflavin does not only serve as photosensitizer but also as a UV-blocker; (3) the UV-irradiance of $3 \mathrm{~mW} / \mathrm{cm}^{2}$ must be homogenous and must have a wavelength of $370 \mathrm{~nm}$; and (4) the minimal thickness of corneal stroma to be cross-linked must at least 400 micrometer. Fulfilling these criteria a damage of the endothelium, the lens or the retina should not be expected.

\section{REFERENCES}

[1] Spoerl E, Huhle M, Seiler T. Induction of cross-links in corneal tissue. Exp Eye Res 1998; 66: 97-103.

[2] Spoerl E, Seiler T. Techniques for stiffening the cornea. J Refract Surg 1999; 15: 711-3.

[3] Spörl E, Schreiber J, Hellmund K, Seiler T, Knuschke P. Studies on the stabilization of the cornea in rabbits. Ophthalmologe 2000; 97: 203-6.

[4] Wollensak G, Spörl E, Reber F, Pillunat L, Funk R. Corneal endothelial cytotoxicity of riboflavin/UVA treatment in vitro. Ophthalmic Res 2003; 35: 324-8.

[5] Wollensak G, Spoerl E, Wilsch M, Seiler T. Endothelial cell damage after riboflavin-ultraviolet-A treatment in the rabbit. J Cataract Refract Surg 2003; 29: 1786-90.

[6] Wollensak G, Spoerl E, Wilsch M, Seiler T. Keratocyte apoptosis after collagen cross-linking using riboflavin/UVA treatment. Cornea 2004; 23: 43-49.

[7] Wollensak G, Spoerl E, Reber F, Seiler T. Keratocyte cytotoxicity of riboflavin/UVA-treatment in vitro. Eye 2004; 18: 718-722.

[8] Wollensak G. Histological changes in human cornea after crosslinking with riboflavin and ultraviolet A. Acta Opthalmol 2010; 88(2): e17-8.

[9] Wollensak G, Spoerl E, Seiler T. Riboflavin/ultraviolet-A-induced collagen cross-linking for the treatment of keratoconus. Am J Ophthalmol 2003; 135: 620-27.

[10] Spörl E, Raiskup-Wolf F, Pillunat LE. Biophysical principles of collagen cross-linking. Klin Monatsbl Augenheilkd 2008; 225: 131-7.

[11] Spoerl E, Mrochen M, Sliney D, Trokel S, Seiler T. Safety of UVA-riboflavin cross-linking of the cornea. Cornea 2007; 26: 3859.
[12] Baiocchi S, Mazzotta C, Cerretani D, Caporossi T, Caporossi A Corneal cross-linking: riboflavin concentration in corneal stroma exposed with and without epithelium. J Cataract Refract Surg 2009; 35: 893-9.

[13] Hayes S, O'Brart DP, Lamdin LS, et al. Effect of complete epithelial debridement before riboflavin-ultraviolet-A corneal collagen crosslinking therapy. J Cataract Refract Surg 2008; 34: 657-61.

[14] Kampik D, Ralla B, Keller S, Hirschberg M, Friedl P, Geerling G. Influence of corneal collagen crosslinking with riboflavin and ultraviolet-a irradiation on excimer laser surgery. Invest Ophthalmol Vis Sci 2010; 51: 3929-34.

[15] Koller T, Seiler T. Therapeutic cross-linking of the cornea using riboflavin/UVA. Klin Monatsbl Augenheilkd 2007; 224: 700-6.

[16] Matthes R. Guidelines on limits of exposure to ultraviolet radiation of wavelengths between $180 \mathrm{~nm}$ and $400 \mathrm{~nm}$ (incoherent optical radiation). Health Physics 2004; 87: 171-186.

[17] Andreassen TT, Simonsen AH, Oxlund H. Biomechanical properties of keratoconus and normal corneas. Exp Eye Res 1980; 31: 435-41.

[18] Mazzotta C, Balestrazzi A, Traversi C, et al. Treatment of progressive keratoconus by riboflavin-UVA-induced cross-linking of corneal collagen: ultrastructural analysis by Heidelberg Retinal Tomograph II in vivo confocal microscopy in humans. Cornea 2007; 26: 390-7.

[19] Mazzotta C, Traversi C, Baiocchi S, et al. Corneal healing after riboflavin ultraviolet-A collagen cross-linking determined by confocal laser scanning microscopy in vivo: early and late modifications. Am J Ophthalmol 2008; 146: 527-33.

[20] McKegney M, Taggart I, Grant MH. The influence of crosslinking agents and diamines on the pore size, morphology and the biological stability of collagen sponges and their effect on cell penetration through the sponge matrix. J Mater Sci Mater Med 2001; 12: 833-44.

[21] Köhler A. "Gedanken zu einem neuen Beleuchtungsverfahren für mikrophotographische Zwecke". Germany: Zeitschrift für wissenschaftliche Mikroskopie 1893.

[22] Semchishen VA, Mrochen M, Seminogov VN, Panchenko VY, Seiler T. - Light beam shaping and homogenization (LSBH) by irregular microlens structure for medical applications. SPIE Proc 1998; 3251: 28-33

[23] Bueller M, Spoerl E, Seiler T, Mrochen M. UV collagen crosslinking of the cornea - safety aspects and design of a UV illumination system. Progress in Biomedical optics and Imaging Proc SPIE 2008; 6844: 1-11

[24] Caporossi A, Baiocchi S, Mazzotta C, et al. Parasurgical therapy for keratoconus by riboflavin-ultraviolet type A rays induced crosslinking of cerneal collagen: preliminary refractive results in an Italian study. J Cataract Refract Surg 2006; 32: 837-45.

[25] Mazzotta C, Traversi C, Baiocchi S, et al. Conservative treatment of keratoconus by riboflavin-uva-induced cross-linking of corneal collagen: qualitative investigation. Eur J Ophthalmol 2006; 16: 530-5.

[26] Wittig-Silva C, Whiting M, Lamoureux E, Lindsay RG, Sullivan LJ, Snibson GR. A randomized controlled trial of corneal collagen cross-linking in progressive keratoconus: preliminary results. J Refract Surg 2008; 24: S720-5.

[27] Vinciguerra P, Albè E, Trazza S, et al. Refractive, topographic, tomographic, and aberrometric analysis of keratoconic eyes undergoing corneal cross-linking. Ophthalmology 2009; 116: 36978 . 